\title{
Intoxicación alimentaria por Clostridium perfringens en el Centro Penitenciario de Atención Institucional de San José. Estudio de cohorte retrospectivo.
}

\author{
Roy Wong-Mc Clure ',Armando Silva-Solórzano 2, Xiomara Badilla-Vargas'
}

\section{$\mathrm{R}$ e s u m e $\mathrm{n}$}

1. Programa de Análisis y Vigilancia Epidemiológica, Caja Costarricense de Seguro Social.

2. Jefe Servicios de Salud, Centro de Atención Institucional de San José

Abreviaturas: INCIENSA, Instituto costarricense de investigación en nutrición y salud; $R R=$ riesgo relativo, IC95\% ; Intervalo de confianza al 95\%;CPE, Clostridium perfringens enterotoxigénica.

Correspondencia: Dr. Roy Wong Mc Clure. E-mail:rwong@ ccss.sa.cr. Fax: 223-II28. Apdo. 4060-1000 San José-Costa Rica. AC

ISSN 0001-6002/2004/46/2/78-83 Acta Médica Costarricense,(C2004 Colegio de Médicos y Cirujanos
Las enfermedades transmitidas por los alimentos se presentan anualmente en Costa Rica y por lo general se estudian hasta identificar el agente etiológico, pero no hasta determinar su fuente de contaminación. No se ha documentado la importancia de fortalecer la inocuidad de alimentos, lo cual produce eventos que comprometen la salud pública cada año, por lo que fue importante estudiar el presente brote.

En este estudio se analiza el brote diarreico de un centro penitenciario de San José, en 2003, procurando determinar el probable alimento contaminado.

Materiales y métodos: El presente estudio corresponde a una cohorte retrospectiva que identifica exposición a los alimentos preparados dentro del Centro, en las 72 horas previas al pico epidémico. La descripción de la cohorte y el cálculo del (RR) se realizó posteriormente para determinar la posible asociación causal. Las muestras de heces fueron procesadas en el laboratorio de referencia delINCIENSA.

Resultados: La totalidad de los individuos pertenecían al sexo masculino, todos privados de libertad, con un promedio de edad de 31.1 años (IC95\%=28.9-33.4). La sintomatología más frecuente fue la diarrea $(92.3 \%)$ y dolor abdominal tipo cólico (84.6\%). El resultado de laboratorio aisló como agente etiológico el Clostridium perfringens enterotoxigénico (CPE).

Al analizar los alimentos suministrados, pico epidémico y período de incubación, se tiene que el salchichón ( $\mathrm{RR}=1.1$;C95\%0.5-2.7) y la preparación con carne molida ( $\mathrm{RR}=2.1, \mathrm{IC} 95 \%: 0.6-7.4)$ mostraron ser los únicos alimentos capaces de transmitir el CPE.

Discusión: El brote del Centro evidencia que la sintomatología descrita corresponde con lo documentado para la intoxicación por CPE y que, tomando en cuenta las condiciones necesarias para la transmisión, solo el salchichón y la preparación con carne molida cuentan con esta plausibilidad biológica. Al considerar el periodo de incubación, los valores de riesgo relativo calculados y la potencia estadística, se concluye que el alimento probablemente contaminado fue la preparación realizada con carne molida, por lo que se deben de reforzar las medidas de control sanitario.

Descriptores: Clostridium perfringens, brote diarréico, enfermedad transmitida por alimentos.

Recibido: 12 de enero de 2004

Aceptado: 23 de marzo de 2004

Un brote transmitido por alimentos se define como un incidente en el cual dos o más personas experimentan una enfermedad similar, resultado de la ingestión de un alimento en común. Antes de 1992 existían tres excepciones a esta definición: intoxicación por botulismo, intoxicación química o por toxina marina, y solo eran consideradas como de transmisión por alimentos si se identificaba el agente etiológico. Desde los principios de 1992, si dos o más 
personas desarrollan la enfermedad entonces son clasificadas dentro de este grupo ${ }^{1}$.

Al igual que los brotes producidos por alimentos, se documentan en la bibliografía otros ocasionados por la contaminación del agua de consumo, o la destinada para fines de recreo, aún cuando estas hayan sido sometidas a cloración o filtración ${ }^{2}$.

En muchas naciones, incluyendo las de alto nivel sanitario, más de la mitad de todos los brotes de enfermedades transmitidas por los alimentos, son causados por contaminación de la carne ${ }^{3}$ o productos cárnicos, que son los que más se asocian con la tóxico-infección producida por Clostridium perfringens, pues ofrecen condiciones ideales para su desarrollo ${ }^{4}$.

El Clostridium perfringens es un germen común responsable de brotes transmitidos por alimentos, especialmente cuando lo que se cocina implica la carne o productos derivados de esta ${ }^{5}$.

El Clostridium perfringens es un bacilo Gram positivo, esporulado y anaerobio, capaz de crecer rápidamente a $45^{\circ} \mathrm{C}$, y cuyo principal hábitat es el suelo y los intestinos de humanos y de animales ${ }^{6}$. Se ha comprobado que esta especie causa una variedad de enfermedades de diferente severidad 7,8 , entre las que se encuentran las de origen alimentario, en virtud de la producción de una enterotoxina, que es una de las 13 toxinas producidas por Clostridium perfringens ${ }^{7,8}$.

El Centro para Control y Prevención de enfermedades EEUU (CDC) ha estimado que el $97 \%$ de los brotes ocurridos por Clostridium perfringens, han tenido como factor contribuyente la inadecuada preparación de los alimentos, o por inapropiadas temperaturas de mantenimiento de estos 9 Se recomienda que para evitar la enfermedad producida por este germen, se sirvan los alimentos calientes o se recalienten hasta alcanzar una temperatura interna mayor o igual a los $74-{ }^{\circ} \mathrm{C}$, antes de servirse ${ }^{9}$.

Según Rodríguez y colaboradores, en Costa Rica se ha aislado el Clostridium perfringens en las plantas procesadoras de carne, tanto en las zonas rurales como urbanas, en el $88 \%$ de las muestras de los suelos aledaños a los establecimientos, en el $93 \%$ de los intestinos de los animales sacrificados, en el $61 \%$ de las carnes procesadas y molidas listas para su distribución, en el $75 \%$ de los expendios de alimentos de las muestras de carne molida y el $36 \%$ de carne en trozo evaluadas ${ }^{4}$. Estos resultados concuerdan con lo descrito por Morera y colaboradores, al estudiar embutidos de plantas procesadoras del área metropolitana de Costa Rica ${ }^{10}$.

Según Gutiérrez y colaboradores, en Costa Rica se ha logrado determinar que el Clostridium perfringens se encuentra en el $63 \%$ de las muestras de carne cocida obtenidas de los establecimientos de alimentación pública, donde se identificaron 37 cepas de este germen de las cuales 12 resultaron positivas, como productoras de enterotoxina ${ }^{11}$. En el perfil epidemiológico de Costa Rica no existe documentación sobre la carga de la enfermedad en la salud pública de eventos relacionados con este agente etiológico.

El sistema Penitenciario costarricense ha sufrido diversos brotes, principalmente de diarreas, entre sus internos, que en ocasiones han comprometido los funcionarios de estos centros.

El Centro de Atención Integral de San José es un centro penitenciario, que se encuentra divido en módulos y estos, a su vez, en cubículos; cada módulo alberga aproximadamente entre 80 a 90 privados de libertad. El 12 de marzo de 2003 se notifica, al Ministerio de Salud por parte del centro médico penitenciario un aumento en el número de casos de diarrea en el Módulo A de dicho centro.

El presente estudio evaluó la etiología y alimento contaminado del brote diarreico ocurrido en el Centro de Atención Institucional de San José, con los alimentos preparados en la cocina de dicho centro durante el período comprendido entre el 10 y el 11 de marzo de 2003.

\section{Materiales y métodos}

Para realizar el estudio se utilizó el cuestionario estandarizado y validado para el estudio de brotes diarreicos del Instituto Nacional de Investigación y Enseñanza en Salud y Nutrición (INCIENSA). Este mismo instituto brindó el análisis respectivo de las muestras biológicas recolectadas dentro de los privados de libertad afectados. Las muestras biológicas de heces fueron procesadas para la determinación del posible agente etiológico, por medio de la aglutinación con látex para la detección de la enterotoxina del Clostridium perfringens.

La digitación de la información y el procesamiento de la misma se realizó con el software EPI INFO 2002, en su versión de enero de 2003, en la primera etapa del análisis; posteriormente se realizó el análisis estadístico con el software Stadistical Package for the Social Science Versión 11.0, definiendo por punto crítico un alpha menor de $0.05(\alpha<0.05)$, como estadísticamente significativo ${ }^{12-15}$.

\section{Diseño de estudio, evaluación de la exposición y cri- terios de inclusión.}

El diseño del estudio corresponde a una cohorte retrospectiva desarrollada en el Centro de Atención Integral de San José, dentro del módulo A del centro.

Se define como la exposición a evaluar todos los alimentos distribuidos y preparados en el Centro, durante los tres días previos a la notificación de los casos, por parte de la oficina de salud del centro.

Se establece como criterio de inclusión a aquellos individuos recluidos en el Centro de Atención Integral de San 
José, en el módulo A, que hubiesen ingresado a este antes del 10 de marzo de 2003, y cuya permanencia se extendiera más allá del 12 de marzo del mismo año. Los alimentos que fueron brindados a los privados de libertad en más de una ocasión fueron denominados según la primera o segunda ingesta según el orden cronológico de reparto.

Se excluyen los privados de libertad que no se encontraban en el centro penitenciario en el periodo de distribución de los alimentos estudiados; obteniendo finalmente un total de 52 individuos que cumplían con los criterios previamente citados.

Resultados
Cuadro1. Distribución de síntomas en población afectada según definición de caso. Centro de Atención Institucional de San José. Marzo, 2003

\begin{tabular}{lcc|}
\hline Síntomas & Número & Porcentaje \\
\hline Diarrea & 24 & 92,3 \\
Calambres & & \\
(dolor abdominal tipo cólico) & 22 & 84,6 \\
Tenesmo & 16 & 61,5 \\
Cefalea & 15 & 57,7 \\
Polimialgias & 10 & 38,5 \\
Náuseas & 8 & 30,8 \\
Fiebre & 6 & 23,1 \\
Vómitos & 5 & 19,2 \\
\hline
\end{tabular}

La media de edad en la cohorte estudiada es de 31.19 años (IC95\%=28.9-33.4), todos del sexo masculino. El grupo expuesto $(\mathrm{n}=)$ cuenta con una media de edad de 29.3 años (IC95\%=28.9-33.4) y el grupo no expuesto $(n=)$ de 33.7 años (IC95\%=28.9-33.4), sin demostrar diferencias estadísticamente significativas $(\mathrm{p}=0.96)$. De los 650 privados de libertad que tiene capacidad para albergar el penal, sólo 26 internos del módulo A (que alojaba a 129 individuos), desarrollaron algún síntoma gastrointestinal. Los síntomas más importantes corresponden a diarrea, la cual se presentó en el 92,3\% de los casos; seguida por espasmos intestinales indicados como dolor abdominal tipo cólico, presentes en el 84,6\% de los casos; el tenesmo en un $61,5 \%$ y la cefalea, en poco más de la mitad de los afectados. Solo una cifra cercana al $20 \%$ desarrolló fiebre y vómitos (Cuadro 1).

Al realizar la curva de tendencia, según la fecha de inicio de los síntomas del grupo afectado, se evidencia que estos iniciaron entre el medio día del 11 de marzo de 2003 y las 4 p m del 13 de marzo de 2003, con un pico epidémico entre las últimas horas del 11 del marzo y las primeras horas del 12 de marzo de 2003.

De los 26 casos que desarrollaron la enfermedad se tomaron 6 muestras de heces para ser procesadas por el laboratorio de referencia INCIENSA, donde se determinó Clostridium Perfringens productora de enterotoxina en dos de ellas.

Todos los afectados identificados recibieron atención ambulatoria y ninguno requirió antibióticoterapia durante el periodo de estudio.

Tomando en cuenta el lapso de incubación del agente aislado y el inicio de la sintomatología de los afectados, se estudió la exposición a los alimentos producidos en la cocina del Centro, desde el 10 de marzo a las 12 medio día hasta el 11 de marzo a las 6 a.m., este periodo incluyó un almuerzo, una cena y un desayuno (Figura 1).

Al contemplar el periodo de interés, se realizó la evaluación de la exposición de los alimentos consumidos dentro
Cuadro 2. Distribución de alimentos según exposición. Centro de Atención Institucional de San José. Marzo, 2003

\begin{tabular}{lcccccc} 
Alimento & \multicolumn{2}{c}{ Expuestos } & No expuestos & \multicolumn{2}{c}{ Total } \\
\hline & No & $\%$ & No & $\%$ & No & $\%$ \\
\hline Ensalada de Lechuga & & & & & & \\
con zanahoria & 40 & 76.9 & 12 & 23.1 & 52 & 100 \\
Papas con salchichón & 42 & 80.8 & 10 & 19.2 & 52 & 100 \\
Arroz primera ingesta & 47 & 90.4 & 5 & 9.6 & 52 & 100 \\
Frijoles primera ingesta & 45 & 86.5 & 7 & 13.5 & 52 & 100 \\
Preparación en salsa & & & & & & \\
con carne molida & 44 & 86.6 & 8 & 15.4 & 52 & 100 \\
Arroz segunda ingesta & 48 & 92.3 & 4 & 7.7 & 52 & 100 \\
Frijoles segunda ingesta & 46 & 88.5 & 6 & 11.5 & 52 & 100 \\
Café segunda ingesta & 38 & 73.1 & 14 & 26.9 & 52 & 100 \\
Pan segunda ingesta & 42 & 80.0 & 10 & 19.2 & 52 & 100 \\
\hline
\end{tabular}

del lapso de incubación, se determinó que una proporción superior al $75 \%$ de los individuos se expusieron a las comidas preparadas en la cocina del centro. Se evidenció que el arroz, los frijoles y la preparación en salsa con carne molida fueron los alimentos con más amplio consumo en los individuos seleccionados (Cuadro 2).

Al analizar la exposición a los alimentos en relación con el desarrollo de la enfermedad por medio de la tasa de ataque (\# de enfermos/ \# de expuestos al riesgo), se evidenció que para todos los alimentos varió entre el $52 \%$ para el alimento con menor tasa hasta un $61 \%$.

Al calcular el riesgo relativo de cada uno de los alimentos seleccionados y según el desarrollo o no de sintomatología gastrointestinal se obtiene como único resultado estadísticamente significativo el consumo de pan en la segunda ingesta $(p<0.05)$ y el resto de los alimentos evaluados no evidencian un RR con significancia estadística, Cuadro 3. 


\begin{tabular}{|c|c|c|c|c|}
\hline \multicolumn{5}{|c|}{$\begin{array}{c}\text { Cuadro 3. Determinación de Riesgo Relativo según } \\
\text { exposición a los diversos alimentos preparados } \\
\text { en la cocina del Centro de Atención Institucional } \\
\text { de San José. Marzo, } 2003\end{array}$} \\
\hline Alimento & $\mathrm{n}$ & RR & IC95\% & $p$ \\
\hline $\begin{array}{l}\text { Ensalada de Lechuga } \\
\text { con zanahoria }\end{array}$ & 52 & 2.30 & $0.833-6.35$ & $0.097^{\mathrm{a}}$ \\
\hline Papas con salchichón & 52 & 1.19 & $0.520-2.710$ & $0.720^{\mathrm{a}}$ \\
\hline Arroz primera ingesta & 52 & 2.66 & $0.451-15.66$ & $0.158^{a}$ \\
\hline Frijoles primera ingesta & 52 & 1.87 & $0.561-6.215$ & $0.419^{a}$ \\
\hline $\begin{array}{l}\text { Caracolitos en salsa } \\
\text { con carne molida }\end{array}$ & 52 & 2.18 & $0.638-7.47$ & $0.248^{a}$ \\
\hline Arroz segunda ingesta & 52 & 2.08 & $0.373-11.62$ & $0.610^{a}$ \\
\hline Frijoles segunda ingesta & 52 & 1.56 & $0.79-5.018$ & $0.668^{a}$ \\
\hline Café segunda ingesta & 52 & 2.82 & $1.00-7.96$ & $0.270^{\mathrm{a}}$ \\
\hline Pan segunda ingesta & 52 & 5.95 & $0.912-38.85$ & $0.011^{a}$ \\
\hline
\end{tabular}

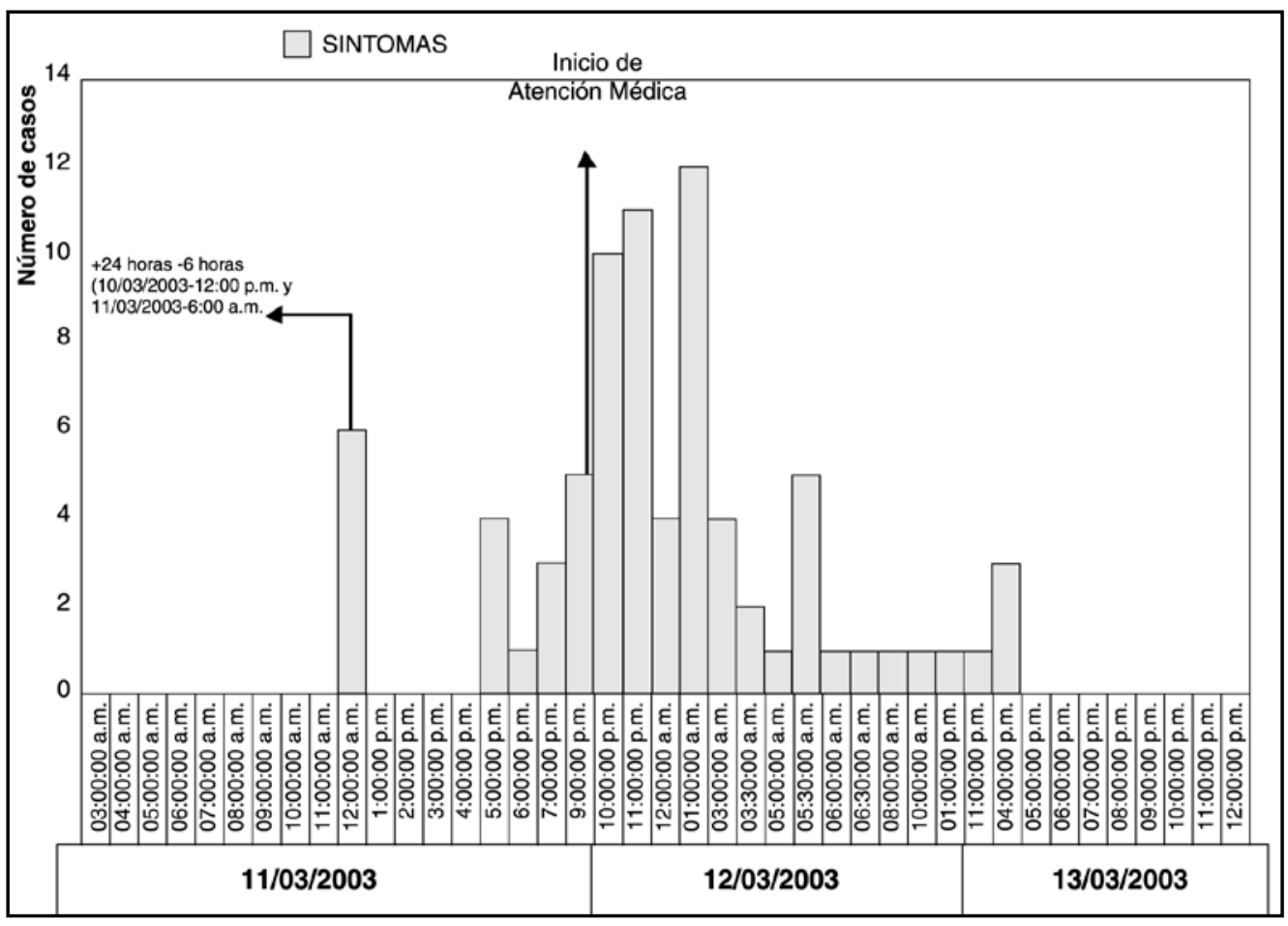

Figura 1. Curva de tendencia según fecha y hora de inicio de síntomas. Centro de Atención Institucional de San José. Costa Rica - Marzo, 2003

$\mathrm{Al}$ realizar el cálculo de la fracción etiológica únicamente para los alimentos con la capacidad biológica de producir la infección se determina que las papas con salchichón cuentan con una fracción etiológica del $15.9 \%$, y de la preparación en salsa con carne molida, del $54.12 \%$.

\section{D i s c u s ión}

La presencia del brote diarreico del centro de atención institucional de San José, ocurrido en marzo de 2003, evidenció una exposición amplia en relación con los alimentos distribuidos a los internos del centro. Sin embargo, la situación epidemiológica se presentó en solamente uno de los módulos constituyentes del Centro, sin comprometer a los funcionarios ni a otros internos en otras secciones, lo cual es probablemente debido a que, en muchas ocasiones, la comida residual de días previos se reparte aleatoriamente en los diversos módulos.

El análisis del perfil sintomático de los afectados evidenció que estaba acorde con el agente etiológico identificado por el laboratorio de referencia en las muestras procesadas.

La curva de tendencia evidenció que se trataba de un brote cuya etiología tuvo una fuente común dentro de los privados de libertad del módulo afectado, y que es, por las características propias del agente etiológico, transmitida por uno de los alimentos que se consumieron durante las horas previas a la hora del inicio de síntomas.

Tomando en consideración el periodo de incubación del Clostridium perfringens y la fecha y hora de inicio de los primero síntomas, se evidencia que la fuente probable de contaminación se encontraba entre las 12 medio día del 10 de marzo y las 6 a.m. el día 11 del mismo mes. Durante este periodo se distribuyó a los privados de libertad un total de nueve alimentos de los cuales solamente el pan brindado a las 6 a.m. del día 11 resultó contar con significancia estadística. ( $R R=5.95$,IC95\% $0.912-38.85, \mathrm{p}<0.05)$ y con la tasa de ataque más elevada.

A pesar de contar con significancia estadística el alimento de la mañana del día 11 de marzo, no cuenta con la plausibilidad biológica para ser el alimento responsable de la transmisión del agente aislado; por esa razón, se identificaron aquellos alimentos con capacidad de ser fuente de 
contaminación para el Clostridium perfringens productor de enterotoxina dentro de los alimentos distribuidos en el periodo definido como máximo de incubación, únicamente se identificaron dos de los alimentos como posible fuente de contaminación por el agente aislado, los cuales fueron las papas con salchichón distribuidas durante el almuerzo del día 10 y la preparación en salsa con carne molida, distribuida en la cena del mismo día. Ninguno de estos alimentos se asoció en forma estadísticamente significativa ( $\mathrm{p}>0.05)$, con la presencia o no de síntomas en la cohorte estudiada.

No obstante, tomando en cuenta la hora de distribución de ambos alimentos y la hora de inicio de los síntomas de la mayoría de los sujetos afectados, resulta altamente improbable que la fuente de contaminación identificada fuesen las papas con salchichón, ya que el periodo trascurrido entre la ingesta y la hora de inicio de los síntomas de la mayoría de la población supera el periodo de incubación por lo que no existe plausibilidad biológica para este alimento.

En relación con la preparación en salsa con carne molida se estima que el tiempo transcurrido entre la hora de distribución de ambos alimentos con la hora de inicio de los síntomas en la mayoría de la población afectada, se encuentra dentro del período de incubación, cuenta con las condiciones necesarias para la transmisión del agente etiológico y además cuenta con la determinación del riesgo relativo más elevada con el intervalo de confianza más amplio, lo cual es concordante con la falta de potencia estadística producto del tamaño muestral.

Al identificar a la preparación en salsa con carne molida como alimento responsable, la fracción etiológica para este alimento es del 54.12\%, lo cual sería la proporción de enfermos que se evitarían si este alimento no hubiese estado contaminado.

Considerando el período de incubación de la enfermedad producida por el Clostridium perfrigens, el tipo alimento brindado y la hora en la que fue distribuido, aunado a la sintomatología asociada y la condición de Costa Rica en relación la contaminación de carnes y sus derivados, los resultados son concordantes con la identificación etiológica brindada por el laboratorio de referencia INCIENSA.

Con los hallazgos encontrados en el presente estudio y los antecedentes del país en relación con el agente aislado y su contaminación en las carnes y embutidos, ya bien estudiados y documentados por el Facultad de Microbiología de la Universidad de Costa Rica, se deben establecer medidas orientadas a una más rigurosa vigilancia epidemiológica de las diarreas e inocuidad de los alimentos.

Es importante establecer un control más estricto en el procesamiento de los productos cárnicos tanto en el ámbito de las plantas destinadas para este propósito como en la preparación de los alimentos. Además, es pertinente que los establecimientos encargados de brindar, como parte de su atención, alimentos a los funcionarios o internos del centro, deben de contemplar en la solicitud de los productos cárnicos la certificación sanitaria de encontrarse libres de Clostridium perfringens y otros agentes potencialmente patógenos al ser humano.

Abstract

Introduction: The sicknesses transmitted by foods appear annually in Costa Rica. They are usually studied until the etiologic agent is identified and but not until the source of contamination is determined. In Costa Rica there are no records of the importance of improving the inocuence of foods by the events that compromise the public health each year therefore the importance to study the present outbreak.

In the present study a diarrheic outbreak in a penitentiary center of San José in the 2003, trying to determine the possible contaminated food.

Materials and methods: The present retrospective cohort study identify as exposition the foods prepared in the center within the 72 hours previous to the epidemic peak. The present study corresponds to a retrospective cohort that identify as exposition to prepared foods within the Center in the previous 72 hours to peak epidemic. The description of the cohort and the calculus of the RR was made posteriously to determine the possible casual association. The samples of feces were processed in the laboratory of reference INCIENSA.

Results: The totality of the individuals were sex male, all inmates, average age 31.19 years $($ CI95\%=28.92-33.46)

The most frequent symptoms are diarrhea (92.3\%) and abdominal colic pain (84.6\%). Laboratory results isolated as etiologic agent the Clostridium perfringens entorotoxigenic (CPE).

Analyzing foods supplied, epidemic peak and incubation period, we have sausage $(\mathrm{RR}=1.19, \mathrm{CI} 95 \% 0.520-2.710)$ and the preparation of grind meat $(\mathrm{RR}=2.18, \mathrm{CI} 95 \%: 0.638-7.47)$, show to be the only food to transmit the CPE.

Discussion: The outbreak in the center evidence that the symptoms described correspond with documentation for intoxication by $\mathrm{CPE}$ and taking in consideration only necessary conditions for the transmission only with sausage and preparation of grind meat, counting with this biologic plausibility.

Considering incubation period, calculated relative risks and the statistics power, we conclude that the probable contaminated food was the preparation made with grind meat, so that sanitary measures must be reinforced. 


\section{Referencias}

1. MMWR Surveillance Summaries. Guidelines for Confirmation for Foodborne-Disease Outbreaks. MMWR;1996; 45: 58-66.

2. MMWR Surveillance for Water borne disease outbreaks-United States, 1993-1994, 1996, 45:1-33.

3. Hatakka M. Microbiological quality of hot meals served by airlines. J Food Prot 1998; 61:1052-1056

4. Rodríguez E, Gamboa M, Vargas P. Clostridium perfringens en carnes crudas y cocidas y su relación con el ambiente en Costa Rica. Ach Lat Nut, 2002; 52:2;

5. Zimoma J, Wenderoth T, Snyder A, French R, Halpin TJ, Florance $\mathrm{JE}$ et al. Clostridium perfringens Gastroenteritis Associated with corned beef served at St. Patrick's Day Meals-Ohio, Virginia, 1993, MMWR; 1994; 43:137-138,143-144.

6. Hatheway CL. Toxigenic Clostridia. Clin Microbiol Rev 1990;3:6698

7. Rood JI. Virulence genes of Clostridium perfringens. Annu Rev Microbiol 1998; 52:333-360.
8. Rood J1, Cole ST, Molecular genetics and pathogenesis of Clostridium perfringens. Microbiol Rev 1991; 55:621-648.

9. Loewenstein MS. Epidemiology of Clostridium perfringens food poisoning. N Engl J Med 1972;286:1026-8.

10. Morera J., Rodríguez E., Gamboa M., Determinación de Clostridium perfringens en embutidos de carne de cerdo del Area Metropolitana de Costa Rica. Ach Lat Nut, 1999; 49:3 .

11. Guitiérrez A. Gamboa M, Rodríguez E, Arias M. Presencia del Clostridium perfringens en preparaciones a base de carne en servicios de alimentación pública del Cantón Central de San José, Costa Rica. Ach Lat Nut, 1999; 49:3

12. Norusis, M. SPSS 11.0 Guide to data Analysis. Prentice Hall. United States, 2000

13. Hazard, B. Statistical Methods for Health Care Research. Lippincott, United States, 2000.

14. Selvin, S. Epidemiologic Analysis. A case oriented approach.. Oxford, United States. 2001

15. Rothman K., Greenland S. Modern Epidemiology. Lippincott-Raven, United States. 1998 